\title{
The structure of the atomic helium trimers: Halos and Efimov states
}

\author{
E. Nielsen, D.V. Fedorov and A.S. Jensen \\ Institute of Physics and Astronomy, \\ Aarhus University, DK-8000 Aarhus C, Denmark
}

April 30, 2019

\begin{abstract}
The Faddeev equations for the atomic helium-trimer systems are solved numerically with high accuracy both for the most sophisticated realistic potentials available and for simple phenomenological potentials. An efficient numerical procedure is described. The large-distance asymptotic behavior, crucial for weakly bound three-body systems, is described almost analytically for arbitrary potentials. The Efimov effect is especially considered. The geometric structures of the bound states are quantitatively investigated. The accuracy of the schematic models and previous computations is comparable, i.e. within $20 \%$ for the spatially extended states and within $40 \%$ for the smaller ${ }^{4} \mathrm{He}$-trimer ground state.
\end{abstract}




\section{Introduction}

Weakly bound two- and three-body systems in low angular momentum states are spatially very extended [1, 2, 3, 国. We shall call them halos, if a substantial fraction of the wave function is outside the range of the potentials. Each of the constituent "particles" may be a composite system, provided it is a tightly bound structure with a binding energy large compared to the interparticle binding energy. Then the corresponding (intrinsic and halo) degrees of freedom decouple and a few-body treatment is appropriate. Prominant examples are the new class of nuclear states called nuclear halos [5, 6] and various molecular systems, in particular the atomic helium trimer 8 , 9].

Halo states are in general characterized by their large spatial extension. They could as well appear as excited states of systems which in the ground state are of a different nature and spatially confined. Borromean systems, where no binary subsystem is bound, are particularly interesting three-body halo candidates [10]. They have by definition a relatively low binding energy. Many nuclear examples are available [2, 5, 6] and until recently it was unclear whether the helium trimer was of Borromean nature [8]. The halo concept is general and of interest in different subfields of physics [1, 2, 3, 4, 5, 6, 7, 8, 9, 10, 11, 12]. In the simplest case of two-body halo systems universal relations between size and binding energy were discussed [1, 13]. Also the general properties of three-body halos are intensively

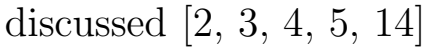

The halo structure is almost entirely determined by the tail properties of the effective interactions between the particles. If the finite-size particles have a substantially overlap, the halo degrees of freedom do not decouple and the fewbody approximations are inaccurate [2, 14]. The effective two-body interactions should then reproduce low-energy scattering properties, i.e. phase shifts or it may be sufficient with the correct scattering length and effective range [2, 14, 15]. For molecular systems it is possible to compute the two-body effective potentials directly from the electromagnetic interaction [8, 16].

The halo structure is technically difficult to compute accurately, since precise knowledge of the behavior of the effective radial (three-body) potential at largedistance is indispensable and at the same time the small distances, responsible for the actual size of the binding energy, must also be properly included. Fortunately, a method treating the large distances analytically and the short distances numerically has recently become available [15, 17, 18, 19]. The method is very powerful as demonstrated by the succesful investigation of the patological Efimov effect [9, 17, 20]. Within the method the Faddeev equations are solved in coordinate space in two steps. First the angular part is solved for each hyperradius, thereby providing the adiabatic basis. This is usually the most difficult part, where semianalytic large-distance asymptotic solutions are employed. Afterwards the coupled set of radial equations for the expansion coefficients is solved.

Another somewhat similar method was recently used to study the helium 
trimer [B]. This method exploit the fact that the interaction (in addition to two distance-coordinates) only depends on one angle, since the three Euler angles describe rotations of the system as a whole. In principle the same adiabatic basis is then obtained. However, the computations only include the lowest angular basis state which already seems to involve extensive calculations.

The ${ }^{4} \mathrm{He}$-dimer and trimer systems have recently been experimentally established as weakly bound systems although without direct measurement of the binding energies [21]. However, the size of the ${ }^{4} \mathrm{He}$-dimer was found to be exceedingly large compared to the range of the two-body potential. In the conceptually simple experiment transmission was measured through a screen consisting of holes with radii comparable to the dimer size [22]. The binding energy of the dimer must consequently be exceedingly small. However, this does not constrain the binding energy of the three-body system which, due to the Thomas-effect [23], still can be much larger than the binding energy of the two-body system. On the other hand, results from solving the Schrödinger equation as well as momentum space Faddeev calculations strongly suggest that the trimer binding energy indeed is very small [8, 24]. Both computations conclude that the trimer has two bound states of which the excited state has the characteristica of an Efimov state: When the attraction is increased it disappears into the continuum away from the discrete three-body spectrum.

Interesting systems can be formed by substituting one or more of the ${ }^{4} \mathrm{He}-$ atoms by ${ }^{3} \mathrm{He}$-atoms. The electron structure is identical and the effective twobody interaction is then unchanged. Due to the smaller masses the three-body states move up in energy and calculations predict that ${ }^{4} \mathrm{He}^{3} \mathrm{He}_{2}$ and the ${ }^{3} \mathrm{He}-$ trimer both are unbound [8]. There is no simple relation between the size and the binding energy of these systems and detailed calculations of the three-body wave functions are needed.

The purpose of the present paper is three-fold. First, we shall repete the investigation of Esry et al. [8] and extent it to include sufficiently many adiabatic basis states to obtain precise solutions. We shall keep an eye on the possible occurrence of Efimov states. Second, we shall define simple two-body potentials reproducing the low-energy scattering behavior of the realistic potentials. We shall assess the accuracy of such approximation schemes, which reduce the necessary computational efforts by orders of magnitude. Third, we shall describe an improved method able to deal efficiently with large repulsive cores as traditionally used in the parametrization of the two-body molecular interaction.

After the introduction we describe first briefly in section 2 the general method of solving the Faddeev equations using hyperspherical coordinates. The angular equations and the effective radial potential at large distances are then analyzed in sections 3 and 4 . The rather delicate numerical procedure and the corresponding results are discussed in sections 5 and 6 , respectively. Finally section 7 contains the summary and the conclusions. 


\section{Theoretical method}

We shall consider an angular momentum zero system of three interacting inert "particles", where the $k$ 'th particle has mass $m_{k}$ and coordinate $\mathbf{r}_{k}$. Their intrinsic degrees of freedom are frozen and only the three-body degrees of freedom shall be treated here. The two-body interactions between the particles $i$ and $j$ are $V_{i j}$. We shall use the three sets of Jacobi coordinates $\mathbf{x}_{i}$ and $\mathbf{y}_{i}$ and the corresponding three sets of hyperspherical coordinates $\left(\rho, \Omega_{i}\right)=\left(\rho, \alpha_{i}, \Omega_{x_{i}}, \Omega_{y_{i}}\right)$ defined by [2, 10]

$$
\begin{aligned}
\mathbf{x}_{i}=\mu_{j k} \mathbf{r}_{j k}, & \mathbf{r}_{j k}=\mathbf{r}_{j}-\mathbf{r}_{k}, \\
\mathbf{y}_{i}=\mu_{i(j k)} \mathbf{r}_{i(j k)}, & \mathbf{r}_{i(j k)}=\mathbf{r}_{i}-\frac{m_{j} \mathbf{r}_{j}+m_{k} \mathbf{r}_{k}}{m_{j}+m_{k}}, \\
x_{i}=\rho \sin \alpha_{i}, & y_{i}=\rho \cos \alpha_{i}, \\
\mu_{j k}=\left(\frac{1}{m} \frac{m_{j} m_{k}}{m_{j}+m_{k}}\right)^{1 / 2}, & \mu_{i(j k)}=\left(\frac{1}{m} \frac{m_{i}\left(m_{j}+m_{k}\right)}{m_{1}+m_{2}+m_{3}}\right)^{1 / 2},
\end{aligned}
$$

where $\{i, j, k\}$ is a cyclic permutation of $\{1,2,3\}$ and $\mu^{2}$ are the reduced masses of the subsystems in units of a normalization mass $m$. The volume element in terms of one of the sets of hyperspherical coordinates is given by $\rho^{5} \mathrm{~d} \Omega \mathrm{d} \rho$, where $\mathrm{d} \Omega=\sin ^{2} \alpha \cos ^{2} \alpha \mathrm{d} \alpha \mathrm{d} \Omega_{x} \mathrm{~d} \Omega_{y}$. The kinetic energy operator is

$$
\begin{array}{r}
T=\frac{\hbar^{2}}{2 m}\left(-\rho^{-5 / 2} \frac{\partial^{2}}{\partial \rho^{2}} \rho^{5 / 2}+\frac{15}{4 \rho^{2}}+\frac{\hat{\Lambda}^{2}}{\rho^{2}}\right), \\
\hat{\Lambda}^{2}=-\frac{1}{\sin (2 \alpha)} \frac{\partial^{2}}{\partial \alpha^{2}} \sin (2 \alpha)+\frac{\hat{l}_{x}^{2}}{\sin ^{2} \alpha}+\frac{\hat{l}_{y}^{2}}{\cos ^{2} \alpha}-4,
\end{array}
$$

where the angular momentum operators $\hat{l}_{x}^{2}$ and $\hat{l}_{y}^{2}$ are related to the $\mathbf{x}$ and $\mathbf{y}$ degrees of freedom.

The total wave function $\Psi$ of the three-body system is written as a sum of three components $\psi^{(i)}$, which in turn for each $\rho$ are expanded on a complete set of generalized angular functions $\Phi_{n}^{(i)}\left(\rho, \Omega_{i}\right)$

$$
\Psi=\sum_{i=1}^{3} \psi^{(i)}\left(\mathbf{x}_{i}, \mathbf{y}_{i}\right)=\frac{1}{\rho^{5 / 2}} \sum_{n} f_{n}(\rho) \sum_{i=1}^{3} \Phi_{n}^{(i)}\left(\rho, \Omega_{i}\right)=\frac{1}{\rho^{5 / 2}} \sum_{n} f_{n}(\rho) \Phi_{n}(\rho, \Omega),
$$

where $\rho^{-5 / 2}$ is the radial phase space factor and $n=1,2,3 \ldots$

The angular functions, the adiabatic basis, are now for each $\rho$ chosen as the eigenfunctions of the angular part of the Faddeev equations:

$$
\frac{\hbar^{2}}{2 m} \frac{1}{\rho^{2}}\left(\hat{\Lambda}^{2}-\lambda_{n}(\rho)\right) \Phi_{n}^{(i)}+V_{j k}\left(\Phi_{n}^{(i)}+\Phi_{n}^{(j)}+\Phi_{n}^{(k)}\right)=0,
$$

where $\{i, j, k\}$ is a cyclic permutation of $\{1,2,3\}$. 
The radial expansion coefficients $f_{n}(\rho)$ are obtained from a coupled set of "radial" differential equations [2], i.e.

$$
\begin{aligned}
\left(-\frac{\partial^{2}}{\partial \rho^{2}}-\frac{2 m\left(E-V_{3}(\rho)\right)}{\hbar^{2}}\right. & \left.+\frac{1}{\rho^{2}}\left(\lambda_{n}(\rho)+\frac{15}{4}\right)-Q_{n n}\right) f_{n}(\rho) \\
& =\sum_{n^{\prime} \neq n}\left(2 P_{n n^{\prime}} \frac{\partial}{\partial \rho}+Q_{n n^{\prime}}\right) f_{n^{\prime}}(\rho)
\end{aligned}
$$

where $E$ is the three-body energy, $V_{3}(\rho)$ is an anticipated additional three-body potential and the functions $P$ and $Q$ are defined as angular integrals:

$$
\begin{gathered}
P_{n n^{\prime}}(\rho) \equiv \int d \Omega \Phi_{n}^{*}(\rho, \Omega) \frac{\partial}{\partial \rho} \Phi_{n^{\prime}}(\rho, \Omega), \\
Q_{n n^{\prime}}(\rho) \equiv \int d \Omega \Phi_{n}^{*}(\rho, \Omega) \frac{\partial^{2}}{\partial \rho^{2}} \Phi_{n^{\prime}}(\rho, \Omega) .
\end{gathered}
$$

Explicit expressions for these coupling terms can be obtained by deriving eq.(8) with respect to $\rho$. By use of the normalization condition $\left\langle\Phi_{n} \mid \Phi_{n^{\prime}}\right\rangle \equiv \delta_{n n^{\prime}}$. we obtain

$$
\begin{aligned}
& P_{n n}=0, P_{n n^{\prime}}=-\frac{\left\langle\Phi_{n}\left|\frac{\partial\left(\rho^{2} v\right)}{\partial \rho}\right| \Phi_{n^{\prime}}\right\rangle}{\lambda_{n}-\lambda_{n^{\prime}}} \text { for } n \neq n^{\prime} \\
& Q_{n n}=\sum_{m \neq n} P_{n m} P_{m n} \\
& Q_{n n^{\prime}}=\sum_{m \neq n, m \neq n^{\prime}} \frac{\lambda_{n}-\lambda_{m}}{\lambda_{n}-\lambda_{n^{\prime}}} P_{n m} P_{m n^{\prime}} \\
& -\frac{\left\langle\Phi_{n}\left|\frac{\partial^{2}\left(\rho^{2} v\right)}{\partial^{2} \rho}\right| \Phi_{n^{\prime}}\right\rangle+2\left(\left\langle\Phi_{n}\left|\frac{\partial\left(\rho^{2} v\right)}{\partial \rho}\right| \Phi_{n}\right\rangle-\left\langle\Phi_{n^{\prime}}\left|\frac{\partial\left(\rho^{2} v\right)}{\partial \rho}\right| \Phi_{n^{\prime}}\right\rangle\right) P_{n n^{\prime}}}{\lambda_{n}-\lambda_{n^{\prime}}} \text { for } n \neq n^{\prime}
\end{aligned}
$$

where $v$ essentially is the sum of the three two-body potentials, i.e. $v=\sum_{i=1}^{3} v_{i}$ with $v_{i}(x)=2 m V_{j k}\left(x / \mu_{j k}\right) / \hbar^{2}$. Thus $P$ is antisymmetric.

\section{Large-distance angular solution}

The angular functions $\Phi_{n}^{(i)}\left(\rho, \Omega_{i}\right)$ are expanded in products of the three-body spherical harmonics $Y_{\ell_{x} m_{x}}\left(\Omega_{x_{i}}\right)$ and $Y_{\ell_{y} m_{y}}\left(\Omega_{y_{i}}\right)$ where the orbital angular momenta and their projections associated with $\mathbf{x}$ and $\mathbf{y}$ are $\left(\ell_{x}, m_{x}\right)$ and $\left(\ell_{y}, m_{y}\right)$. The total angular momentum is zero and we get

$$
\Phi_{n}^{(i)}\left(\rho, \Omega_{i}\right)=\sum_{\ell} \frac{\phi_{n \ell}^{(i)}\left(\rho, \alpha_{i}\right)}{\sin \left(2 \alpha_{i}\right)}\left[Y_{\ell m}\left(\Omega_{x_{i}}\right) \otimes Y_{\ell-m}\left(\Omega_{y_{i}}\right)\right]^{00}
$$


where $\sin \left(2 \alpha_{i}\right)$ is a factor related to phase space. We can now insertion of eq. (15) into eq.(8) and obtain an equation for each of $\ell$ projections of each Faddeev component:

$$
\begin{array}{r}
\left(-\frac{\partial^{2}}{\partial \alpha_{i}^{2}}+\frac{\ell(\ell+1)}{\sin ^{2} \alpha_{i}}+\frac{\ell(\ell+1)}{\cos ^{2} \alpha_{i}}+\rho^{2} v_{i}\left(\rho \sin \alpha_{i}\right)-\nu_{n}^{2}(\rho)\right) \phi_{n \ell}^{(i)}\left(\rho, \alpha_{i}\right)= \\
-\rho^{2} v_{i}\left(\rho \sin \alpha_{i}\right) R_{\ell}^{(i)}\left(\alpha_{i}\right)
\end{array}
$$

where $R_{\ell}^{(i)}$ is the sum of the other two Faddeev components projected onto $\left[Y_{\ell m}\left(\Omega_{x_{i}}\right) \otimes Y_{\ell-m}\left(\Omega_{y_{i}}\right)\right]^{00}$ in the $i$ 'th Jacobyan system. I.e. $R_{\ell}^{(i)}$ is a sum of all the non-zero $\ell$-contributions of the two other Faddeev components. A detailed analysis shows that $R_{\ell}^{(i)}\left(\alpha_{i}\right)$ is proportional to $\sin ^{\ell+1} \alpha_{i}$.

When $\rho$ is large the potential is zero everywhere except for small $\alpha_{i}$. Thus for $\ell>0$ the effect of the potential on the left hand side of the equation have to been seen through a centrifugal barrier proportional to $\ell(\ell+1)$ and the effect of the potential on the right hand side is quenched by a factor of $\alpha_{i}^{1+\ell}$.

Thus for non-zero $\ell$ all terms containing the potential can be put to zero for large enough $\rho$. Thus the free equation appear. So if any of the Faddeev components have a finite contribution with $\ell>0$ for $\rho \rightarrow \infty$ we obtain the free solution for that component with the free eigenvalue $\nu_{n}^{2}=(K+2)^{2}$ where $K$ is an even integer equal or greater than $2 \ell$. Again a detailed analyses shows that $\nu_{n}^{2}=(K+2)^{2}+O\left(\rho^{-1-2 \ell}\right)$. This analysis is actually quite similar to the ones given below except higher transcendental functions have to be used in the derivation.

If we now assume that all the Faddeev components only have contributions from $\ell=0$ for $\rho \rightarrow \infty$ we end up with three coupled asymptotic equations

$$
\begin{gathered}
\left(\frac{\partial^{2}}{\partial \alpha_{i}^{2}}-\rho^{2} v_{i}\left(\rho \sin \alpha_{i}\right)+\nu^{2}(\rho)\right) \phi_{0}^{(i)}\left(\rho, \alpha_{i}\right)=\rho^{2} v_{i}\left(\rho \sin \alpha_{i}\right) \\
\times \sum_{j \neq i} \frac{1}{\sin \left(2 \varphi_{k}\right)} \int_{\left|\varphi_{k}-\alpha_{i}\right|}^{\pi / 2-\left|\pi / 2-\varphi_{k}-\alpha_{i}\right|} d \alpha_{j} \phi_{0}^{(j)}\left(\rho, \alpha_{j}\right) \\
\tan \varphi_{k}=\sqrt{\frac{m_{k}\left(m_{i}+m_{j}+m_{k}\right)}{m_{i} m_{j}}} .
\end{gathered}
$$

The short-range potential $v_{i}\left(\rho \sin \alpha_{i}\right)$ can be assumed to vanish for $\alpha_{i}$ larger than a constant $\alpha_{0}^{(i)}$ which for large distances must decrease inversely proportional to $\rho$. The corresponding solution to eq.(17) is then

$$
\phi_{0}^{(i)}\left(\rho, \alpha_{i}\right)=A_{0}^{(i)} \sin \left[\nu\left(\alpha_{i}-\frac{\pi}{2}\right)\right] \text { for } \alpha_{i}>\alpha_{0}^{(i)},
$$

where $A_{0}^{(i)}$ is an arbitrary constants and the proper boundary condition $\phi_{0}^{(i)}(\rho, \alpha=$ $\pi / 2)=0$ explicitly is included. 
The function $\phi_{0}^{(j)}\left(\rho, \alpha_{j}\right)$ in eq.(17) is only needed when $v_{i}\left(\rho \sin \alpha_{i}\right)$ remains finite, i.e. for $\alpha_{i}<\alpha_{0}^{(i)}$. When $\alpha_{0}^{(i)}+\alpha_{0}^{(j)}<\varphi_{k}$ the integration in eq.(17) only includes the region where eq.(17) is valid. Therefore as soon as $\rho$ corresponds to distances outside the short-range potentials we can rewrite eq.(17) as

$$
\begin{aligned}
&\left(\frac{\partial^{2}}{\partial \alpha_{i}^{2}}-\rho^{2} v_{i}\left(\rho \sin \alpha_{i}\right)+\nu^{2}(\rho)\right) \phi_{0}^{(i)}\left(\rho, \alpha_{i}\right)=2 \rho^{2} v_{i}\left(\rho \sin \alpha_{i}\right) \frac{\sin \left(\nu \alpha_{i}\right)}{\nu} \\
& \times \sum_{j \neq i} A_{0}^{(j)} \frac{\sin \left(\nu\left(\varphi_{k}-\frac{\pi}{2}\right)\right)}{\sin \left(2 \varphi_{k}\right)} .
\end{aligned}
$$

The solution to eq.(20) is given in eq.(19) for $\alpha_{i}>\alpha_{0}^{(i)}$ and for $\alpha_{i}<\alpha_{0}^{(i)}$ we find

$$
\phi_{0}^{(i)}\left(\rho, \alpha_{i}\right)=\phi_{0 h}^{(i)}\left(\rho, \alpha_{i}\right)-\frac{2}{\nu} \sin \left(\nu \alpha_{i}\right) \sum_{j \neq i} \frac{A_{0}^{(j)} \sin \left(\nu\left(\varphi_{k}-\frac{\pi}{2}\right)\right)}{\sin \left(2 \varphi_{k}\right)},
$$

where $\phi_{0 h}^{(i)}$ is the solution to the homogeneous equation

$$
\left(\frac{\partial^{2}}{\partial \alpha_{i}^{2}}-\rho^{2} v_{i}\left(\rho \sin \alpha_{i}\right)+\nu^{2}(\rho)\right) \phi_{0 h}^{(i)}\left(\rho, \alpha_{i}\right)=0
$$

with the boundary conditions $\phi_{0 h}^{(i)}\left(\rho, \alpha_{i}=\frac{\pi}{2}\right)=\phi_{0 h}^{(i)}\left(\rho, \alpha_{i}=0\right)=0$. If $v_{i}\left(\rho \sin \alpha_{i}\right)$ varies within the interval $\alpha_{i}<\alpha_{0}^{(i)}$ the solution to eq.(22) could be complicated and only obtained numerically. However, if $v_{i}(x)$ approaches a finite constant for small $x$ we find immediately

$$
\phi_{0 h}^{(i)}\left(\rho, \alpha_{i}\right)=B_{0}^{(i)} \sin \left(\alpha_{i} \sqrt{\nu^{2}(\rho)-\rho^{2} v_{i}(0)}\right)
$$

for arbitrary constants $B_{0}^{(i)}$.

Assuming that $\nu \alpha_{0}^{(i)}$ is small for large $\rho$ we can expand the right hand side of eq.(17) to leading order in $\alpha_{i}$. This gives

$$
\begin{gathered}
\left(\frac{\partial^{2}}{\partial \alpha_{i}^{2}}-\rho^{2} v_{i}\left(\rho \sin \alpha_{i}\right)+\nu^{2}(\rho)\right) \phi_{0}^{(i)}\left(\rho, \alpha_{i}\right)=2 \alpha_{i} C^{(i)} \rho^{2} v_{i}\left(\rho \sin \alpha_{i}\right) \\
C^{(i)} \equiv \frac{\phi_{0}^{(j)}\left(\rho, \varphi_{k}\right)}{\sin \left(2 \varphi_{k}\right)}+\frac{\phi_{0}^{(k)}\left(\rho, \varphi_{j}\right)}{\sin \left(2 \varphi_{j}\right)}
\end{gathered}
$$

The eigenvalue solutions $\nu_{n}^{2}$ to eqs.(17), (20) and (24) converge towards the hyperspherical spectrum as $\rho$ increases. Due to the coupling the asymptotic values are now approached over a distance defined by the scattering lengths, which might be very much larger than the ranges of the interactions.

The potentials $\rho^{2} v_{i}\left(\rho \sin \alpha_{i}\right)$ vanish for large $\rho$ for all $\alpha_{i}$ except in a narrow region around zero. The conditions for the effective range approximation therefore 
become better and better fulfilled as $\rho$ increases and any potential with the same scattering length and effective range would lead to the same results. Let us then in the region of large $\rho$ use square well potentials $V_{j k}(r)=-S_{0}^{(i)} \Theta\left(r<R_{i}\right)$, or equivalently expressed by the reduced quantities $v_{i}(x)=-s_{0}^{(i)} \Theta\left(x<X_{i}=R_{i} \mu_{j k}\right)$, where the parameters are adjusted to reproduce the two-body scattering lengths and effective ranges of the initial potential. The corresponding solutions are then accurate approximations to our original problem at distances comparable to the range of the potentials [19].

Using square well potentials we can now easily define the constants $\alpha_{0}^{(i)}=$ $\arcsin \left(X_{i} / \rho\right)$ such that the potentials $v_{i}\left(\rho \sin \alpha_{i}\right)$ for large $\rho$ are zero in region II where $\alpha_{i}>\alpha_{0}^{(i)}$ and finite and constant in region I where $\alpha_{i}<\alpha_{0}^{(i)} \ll 1$. The square well solutions are then given in eqs.(19), (21) and (23). We can also solve the approximate equation in eq.(24) for both region I and II. We obtain again the same solutions, where $\nu^{2}(\rho)$ is neglected compared to $\rho^{2} v_{i}(0)$ and $\sin \left(\nu \alpha_{i}\right)$ in the last term of eq.(21) should be replaced by $\nu \alpha_{i}$. Matching these solutions at the boundary $\alpha_{i}=\alpha_{0}^{(i)}$ provides the quantization condition and determines the eigenvalue $\nu$.

Also the decoupled equations in eq.(16) for non-zero $\ell$-values can be solved for square well potentials. In region I and II the solutions are proportional to the spherical Bessel functions and the hyperspherical polynomials, respectively.

All these square well solutions coincide at large distances with the solutions for any general potential with the same scattering length and effective range. However, when the two-body potentials are strongly repulsive at small distances it is an advantage to use eq.(20) with the correct potential instead of both the square well solution and the additional approximation in eq.(24). A faster convergence (for smaller $\rho$ ) towards the large-distance asymptotics is then obtained.

The method described here is useful when the large-distance behavior is important. In extreme cases as for example for Efimov states and halo systems the large distances are necessary and otherwise difficult or impossible to compute sufficiently accurate. For large distances, where the approximations become increasingly better, it is in any case much easier to obtain accurate solutions from the asymptotic equations than from the original integro-differential equations in eq.(17). Thus we have formulated a convenient numerical procedure.

\section{The effective potential and the Efimov states}

The most important ingredient in the radial equation is the angular eigenvalue $\lambda=\nu^{2}-4$. We can extract the essential properties by analytical analysis of a schematic model. For simplicity we consider three identical bosons. We first find the square well potential $-S_{0} \Theta\left(r<R_{0}\right)$ with the same scattering length $a_{s}$ and effective range $R_{e f f}$ as the original two-body potential. The lowest-lying adiabatic 
radial potentials are then asymptotically close for the two potentials for hyperradii $\rho$ larger than $2 R_{0}$. The large-distance behavior is essentially determined by the $s$-waves. The resulting eigenvalue equation, derived from eqs.(19), (21) and (23), is a simple transcendental equation [19]

$$
\begin{aligned}
& \frac{\kappa}{\nu} \cos \left(\alpha_{0} \kappa\right)\left[\frac{8}{\sqrt{3}} \sin (\nu \pi / 6) \sin \left(\nu \alpha_{0}\right)-\nu \sin \left(\left(\alpha_{0}-\pi / 2\right) \nu\right)\right] \\
= & \sin \left(\alpha_{0} \kappa\right)\left[\frac{8}{\sqrt{3}} \sin (\nu \pi / 6) \cos \left(\alpha_{0} \nu\right)-\nu \cos \left(\left(\alpha_{0}-\pi / 2\right) \nu\right)\right],
\end{aligned}
$$

where $\kappa=\sqrt{2 m S_{0} \rho^{2} / \hbar^{2}+\nu^{2}(\rho)}$ and $\alpha_{0}=\arcsin \left(R_{0} / \rho \sqrt{2}\right)$.

The lowest-lying hyperradial potential in eq.(9) are to the lowest orders in $1 / \rho$ obtained by expansion of eq.(26). For $R_{0} \ll \rho \ll\left|\mu_{i j} a_{s}\right|$, where $a_{s}$ is the scattering length, we find

$$
\begin{gathered}
\lambda(\rho) \approx \nu_{E}^{2}-4-c_{E} \frac{R_{0} \mu_{i j}}{\rho}\left(1-\frac{\pi^{2}}{48 \nu_{E}^{2}}\right), \\
c_{E}=\frac{\nu_{E}^{3} \sin \left(\frac{\pi}{2} \nu_{E}\right)}{\frac{4 \pi}{3 \sqrt{3}} \cos \left(\frac{\pi}{6} \nu_{E}\right)-\cos \left(\frac{\pi}{2} \nu_{E}\right)+\frac{\pi}{2} \nu_{E} \sin \left(\frac{\pi}{2} \nu_{E}\right)}=0.486214,
\end{gathered}
$$

where $\nu_{e}^{2}=-1.0125$ is the solution to the Efimov equation obtained by expansion of eq.(26) in the limit where $a_{s} / \rho= \pm \infty$, i.e.

$$
8 \sin \left(\nu_{E} \pi / 6\right)=\nu_{E} \sqrt{3} \cos \left(\nu_{E} \pi / 2\right)
$$

In the other limit where $\left|\mu_{i j} a_{s}\right| \ll \rho$ we find instead for the lowest eigenvalue円

$$
\lambda(\rho) \approx-\frac{48 a_{s} \mu_{i j}}{\pi \rho} \text { for } a_{s}>0, \lambda(\rho) \approx-\frac{\rho^{2}}{\mu_{i j}^{2} a_{s}^{2}} \text { for } a_{s}<0 .
$$

The effective radial potential is in this way obtained analytically for the square well potential for various distance intervals when the scattering length is large compared to the interaction range. However, as indicated above these results are more general, since they can be expressed in terms of scattering length and effective range, which are universal quantities characterizing low-energy two-body scattering properties of short-range potentials. For distances smaller than $R_{0}$ the details of the original two-body potentials are essential and the square well results are not applicable. This part of the potential is decisive for the actual (small) binding energy of the three-body system.

Between a few times $R_{0}$ and a few times below $15 \mu_{i j}\left|a_{s}\right|$ eq.(27) is valid. This region could be called the Efimov region, since the Efimov states appear here for $\rho<\left|\mu_{i j} a_{s}\right|$ where the radial potential in eq.(9) behaves as $\rho^{-2}$. The lowest

\footnotetext{
${ }^{1}$ We assume a negative scattering length for a two-body system with one bound state
} 
angular and radial wave functions $\Phi$ and $f_{n}$ are then obtained from eqs.(15), (19) and (9) as

$$
\Phi_{n} \propto \sum_{i=1}^{3} \frac{\sin \left(\nu_{E}\left(\alpha_{i}-\pi / 2\right)\right)}{\sin \left(2 \alpha_{i}\right)}, f_{n} \propto \sqrt{\rho} K_{\nu_{E}}\left(\rho \sqrt{2 m|E| / \hbar^{2}}\right),
$$

where $K_{\nu_{E}}$ is the modified Bessel function with an imaginary index. The radial function could easily be obtained from eq.(9) in the limits where either the energy or the centrifugal barrier term is dominating. The same result is found by expansion of eq. (31) for small and large values of $\rho$

$$
\begin{array}{r}
f_{n} \propto \sqrt{\rho} \sin \left(\left|\nu_{E}\right| \ln \left(\frac{\rho}{\mu_{i j} R_{e f f}}\right)\right) \text { for } \rho^{2}<\hbar^{2} /(2 m|E|) . \\
f_{n} \propto \exp \left(-\rho \sqrt{2 m|E| / \hbar^{2}}\right) \text { for } \rho^{2}>\hbar^{2} /(2 m|E|),
\end{array}
$$

where the zero point for the first oscillation in $\rho$ is assumed to be $\mu_{i j} R_{e f f}$. The exponential fall off at large distance occurs for all bound states for distances larger than $\hbar^{2} /(2 m|E|)$, since the effective radial potential falls off faster than $\rho^{-3}$.

The number of Efimov states is determined by the number of possible oscillations of the sine function within the interval $\rho<\left|15 \mu_{i j} a_{s}\right|$. Each new oscillation corresponds to another further excited state and the zero point for the $k$ 'th oscillation is then given by

$$
\rho_{k} \approx \mu_{i j} R_{e f f} \exp \left(\frac{k \pi}{\left|\nu_{E}\right|}\right) .
$$

The number of Efimov states $\mathrm{N}$ is estimated from eq.(30) by $\rho_{N} \approx\left|15 \mu_{i j} a_{s}\right|$, i.e.

$$
N \approx \frac{\left|\nu_{E}\right|}{\pi} \ln \left(\frac{15\left|a_{s}\right|}{R_{e f f}}\right)
$$

The $k$ 'th state has a radial extension of about $\rho_{k}$, i.e. exponentially increasing with $k$, and correspondingly an exponentially decreasing energy

$$
E_{k} \approx \frac{\hbar^{2}}{2 m R_{e f f}^{2}} \exp \left(-\frac{2 k \pi}{\left|\nu_{E}\right|}\right) \text {. }
$$

Clearly infinitely large $a_{s}$ then gives infinitely many bound Efimov states. They are extremly weakly bound and extremely spatially extended.

Efimov states may also be present in non-identical bosonic three-body systems. The condition is that at least two of the binary subsystems have bound $s$-states at zero energy or equivalently infinitely large scattering lengths. The third binary subsystem must be without bound states. A finite number of states with similar properties appear when the conditions are approximately fulfilled. The appearance is determined by the large-distance behavior of the zero angular momentum states. 


\section{$5 \quad$ Numerical procedure}

It should be straightforward to solve the equations of motion numerically. However, two major difficulties must be tackled. The first is related to the large short-range repulsive core where the wave function must be extremely small. The potential has an attractive pocket at short distances where the wave function also is small. These inner regions are where the potential is relatively large, are decisive for the energy of the bound states and the corresponding wave function must therefore be calculated with high accuracy. The second problem is that the weakly bound states are located far outside the range of the potential and the appropriate long-range part of both the potential and the wave function are therefore also decisive. A marginal numerical inaccuracy could be crucial for the number of loosely bound states.

\subsection{Method}

Our first overall computing strategy is to use the Faddeev decomposition of the Schrödinger equation. The proper boundary conditions and the more subtle correlations are then much easier to account for in actual computations. The second computing strategy is to expand on a suitable angular basis for short distances and solve the large-distance asymptotic angular equations directly at large distances. In an overlapping region these solutions are then combined. Finally the coupled set of radial equations is solved.

For short distances each Faddeev component is then expanded on the hyperspherical basis [12, 10, 2]. This amounts for each $\rho$ to an expansion in eq. (15) of the functions $\phi^{(i)}$ in Jacobi polynomials. The remaining expansion coefficients are functions of $\rho$. The total angular basis then consists of a carefully selected set of hyperspherical harmonics with the total angular momentum zero. The basis is characterized by the Faddeev components $i=1,2,3$, the quantum numbers $\ell=0,1,2,3 \ldots$ and the hyperspherical quantum number $K=0,2,4,6 \ldots$.

Only basis functions with the correct symmetry properties are included, i.e. symmetric or antisymmetric for bosons or fermions. When $\rho^{2} v_{i}$ is small for small $\rho$ the angular eigenvalue equation eq.(8) only includes the kinetic energy operator with the corresponding hyperspherical harmonics as solutions. In this case a few of the lowest $\ell$ and $K$ quantum numbers are sufficient in the basis.

For a repulsive core, where $\rho^{2} v_{i}$ diverges (or simply is very large), larger $\ell$ values are needed in the basis to produce a vanishing wave function at small distances. The angular matrix elements of the potentials become large for small $\rho$ even for high $\ell$, where the contribution usually vanishes due to the centrifugal barrier. A faster convergence in $\ell$ is achieved by subtracting a constant $V_{0}$ from each of the two-body potentials and subsequently correcting the energy eigenvalue by $3 V_{0}$. This is simply a shift of the energy scale by $3 V_{0}$. If the infinite basis is 
used the choice of constant makes no difference. A minimal basis size is obtained when $V_{0}$ approximately equals the lowest angular eigenvalue.

Increasing $\rho$ normally decreases the required maximum $\ell$-value since the centrifugal barrier then increases. On the other hand larger hyperspherical quantum numbers (or polynomial orders) are needed, because for short-range interactions the potentials in $\alpha$-space become increasingly confined to a narrow region of phase space around $\alpha_{i}=0$. Accurate wave functions in this decreasing region around $\alpha_{i}=0$ require high-order Jacoby polynomials (high $K$ ) to reproduce the rapid change caused by the corresponding behavior of the potential.

In the limit of very large $\rho$ the hyperspherical spectrum is also approached for short-range potentials. Therefore in this extreme limit only small $\ell$ and small $K$ are needed to obtain the angular eigenvalue spectrum. However, in the sometimes very extended transition region of intermediate $\rho$-values the eigenvalues are required with high precision. Thus, increasing $\rho$ demands an increasing basis for an accurate description. It is crucial that this basis only needs to include high-order polynomials, but still only small angular momenta. The necessary basis can then be substantially reduced from the full hyperspherical basis with all $K$ and $\ell$ due to the use of the Faddeev equations instead of the Schrödinger equation.

We shall treat large $\rho$ by considering $s$-waves, $\ell=0$, without the further expansion on the Jacobi-polynomia, i.e. we shall solve the large-distance equations (20) directly. Moving towards smaller $\rho$ we must then find an overlap region where the large- and small-distance solutions coincide. To optimize computational speed and accuracy the basis must therefore be carefully selected to provide sufficient accuracy in the overlap region.

When the asymptotic states correspond to $\ell \neq 0$ the convergence is expected to be much faster and essentially reached already at small distance. We extrapolate the $\lambda$-values by using the form $K(K+4)+c \rho^{-2}$ where $c$ is a constant obtained by matching and $K$ is the asymptotic hyperspherical quantum number of this eigenvalue.

The direct way of calculating the coupling terms $P_{n n^{\prime}}$ and $Q_{n n^{\prime}}$ is to perform a numerical derivation, but this numerical double derivative is inaccurate in regions where the wave functions change rapidly, i.e. for repulsive cores at small distances. Instead we used the explicit expressions in eq.(12) for small distances. For large distances we used numerical derivation for the $P_{n n^{\prime}}$ and $Q_{n n}$ and a smooth extrapolation of the small distance functions for the non-diagonal $Q_{n n^{\prime}}$.

\subsection{Parameters}

In the actual cases of the He-trimer systems we choose the mass unit $m$ in eq.(5) in the definition of the Jacobian coordinates [2, 10, 12] to be one nuclear mass unit $m=1822.887$ a.u. (atomic mass unit). The masses of the He-atoms are then $m\left({ }^{4} \mathrm{He}\right)=4.002603250 m$ and $m\left({ }^{3} \mathrm{He}\right)=3.016026 m$. 
The two-body interaction is the LM2M2 potential from [26]. The three-body interaction $V_{3}$ is very small [31] and assumed to be zero in the present computation. For the ${ }^{4}$ He-trimer the effective range of the potential is $R_{e f f}=13.843$ a.u. ( 1 atomic length units $=0.529177 \AA$ ) and the binding energy is estimated to be of the order of $1 \mathrm{mK}\left[8\right.$, 22] (1 Kelvin $=0.0861735 \mathrm{meV}=3.16679 \cdot 10^{-6}$ atomic energy units). This extremely low binding energy is equivalent to a two-body scattering length of $a_{s}=-189.054$ a.u., which is much larger than the effective range. The size of the ${ }^{4} \mathrm{He}$-dimer is measured to be $\left\langle\left|\mathbf{r}_{1}-\mathbf{r}_{2}\right|\right\rangle=120 \pm 20$ a.u. [22]. For the ${ }^{4} \mathrm{He}-{ }^{3} \mathrm{He}$ system the different masses instead give $a_{s}=33.261$ a.u. and $R_{e f f}=18.564$ a.u. and for ${ }^{3} \mathrm{He}-{ }^{3} \mathrm{He}$ we get $a_{s}=13.520$ a.u. and $R_{e f f}=25.717$ a.u.

For the ${ }^{4}$ He-trimer we used a basis set of 150,40 Jacobi polynomials for each Faddeev component for $\ell=0,2$, respectively and 30 for $\ell=4,6,8$. This amounts to 840 basis states in total, but after projecting on the states with correct symmetry the basis size was reduced to 235 . For the ${ }^{3} \mathrm{He}^{4} \mathrm{He}_{2}$ and ${ }^{4} \mathrm{He}^{3} \mathrm{He}_{2}$ molecules we used a basis set of 150,60, 50,45, 40,35,30,25 Jacobi polynomials for each Faddeev component for $\ell=0,1,2,3,4,5,6,7$, respectively and 20 for $\ell=8,9,10,11,12,13,14$. The components related to two identical ${ }^{4} \mathrm{He}$ or ${ }^{3} \mathrm{He}-$ atoms only needs even $\ell$ due to the symmetry requirement. For the asymmetric trimers this amounts to a total basis of 1500 which reduced to 638 after symmetry restoration. In all cases this corresponds to an overlap region between small and large distances around $\rho=100$ a.u. The converged results from the basis diagonalization and the asymptotic solutions are then used below and above 100 a.u., respectively.

The convergence and the accuracy of the numerical results were tested thoroughly by varying the number of Jacobi polynomials in each component as well as the number of partial waves. The results are also independent of relatively small variations of the position of the matching point between the small and large distance solutions. Thus we expect reliable numerical results.

\section{$6 \quad$ Numerical results}

The numerical procedure and the input parameters are now specified. The results are obtained by expansion on the adiabatic basis and we shall therefore first compute the corresponding angular eigenvalues. Then we shall show energies and wave functions for both the LM2M2 and the simple schematic potentials. Finally we discuss the various intrinsic geometric structures with special emphasis on the possible appearence of Efimov states. 


\subsection{Angular eigenvalues}

The decisive quantities are the adiabatic potentials where the main components are the angular eigenvalues. The lowest of these are computed and shown in fig. 1] s functions of $\rho$ for the ${ }^{4} \mathrm{He}$-trimer. Both the general small-distance numerical solutions and the asymptotic large-distance $s$-wave solutions are shown. They diverge for small $\rho$ due to the strongly repulsive core in the interaction between two He-atoms. The lowest of these eigenvalues has an attractive pocket at small distance and diverges parabolically towards $-\infty$ for large $\rho$. The pocket is responsible for the three-body bound states and the divergence reflects the bound state in the two-body subsystem. Due to the very small two-body binding energy the lowest level remains almost constant up to about $1000 \mathrm{a}$.u. where the divergence sets in. The overlap region above which the asymptotic solutions can be used is seen to be around 100 a.u.

The higher lying eigenvalues asymptotically approach the hyperspherical spectrum defined by $K(K+4)$ where $K$ is a non-negative integer. In the present case $K$ must furthermore be an even number because the total orbital angular momentum is zero. The requirement of totally symmetric wave functions removes a number of otherwise possible degenerate states. This eliminates completely all $K=2$ states whereas the asymptotic states corresponding to $K=0,4,6,8,10$ all are non-degenerate and with the angular quantum number $\ell=0$. For $K=12$ the s-wave solution is accompanied by a higher angular momentum solution, which has converged to the asymptotic value $12(12+4)=192$ already at about $\rho=100$ a.u. This level and that of $K=10$ avoided crossing at about $\rho=80$ a.u. The even higher lying levels are also degenerate corresponding to more than one totally symmetric state.

The angular eigenvalue spectrum changes when the asymmetric ${ }^{3} \mathrm{He}^{4} \mathrm{He}_{2}$ molecule is considered, see fig. 2. The interactions are the same as for the symmetric case but the masses are different. The number of levels is roughly increased by a factor of two due to the less restrictive symmetry requirement. The divergence for $\rho=0$, the parabolic divergence at large $\rho$ due to the twobody bound state and the convergence to the hyperspherical spectrum at large distances are still the dominating features. Compared to the ${ }^{4} \mathrm{He}$-system we now find a number of additional levels. They correspond to nearly antisymmetric solutions in the exchange of ${ }^{3} \mathrm{He}$ and ${ }^{4} \mathrm{He}$ whereas the "original" levels also present for the ${ }^{4}$ He-trimer must be of symmetric character.

Considering the ${ }^{4} \mathrm{He}^{3} \mathrm{He}_{2}$-molecule obtained by substituting another ${ }^{4} \mathrm{He}$ by a ${ }^{3}$ He-atom again decreases all the binding energies. We show the angular eigenvalue spectrum in fig. 3, where the usual features are seen. However, now the lowest eigenvalue converges to zero for large $\rho$, since none of the two-body subsystems form bound states. The asymptotic spectrum is now reached much faster than for the two previous cases. This rate of convergence is roughly proportional to the sum of the three scattering lengths or more precisely $\frac{16}{\pi} \sum_{i=1,3} a_{s}^{(i)} \mu_{j k}\left(a_{s}^{(i)}\right.$ 


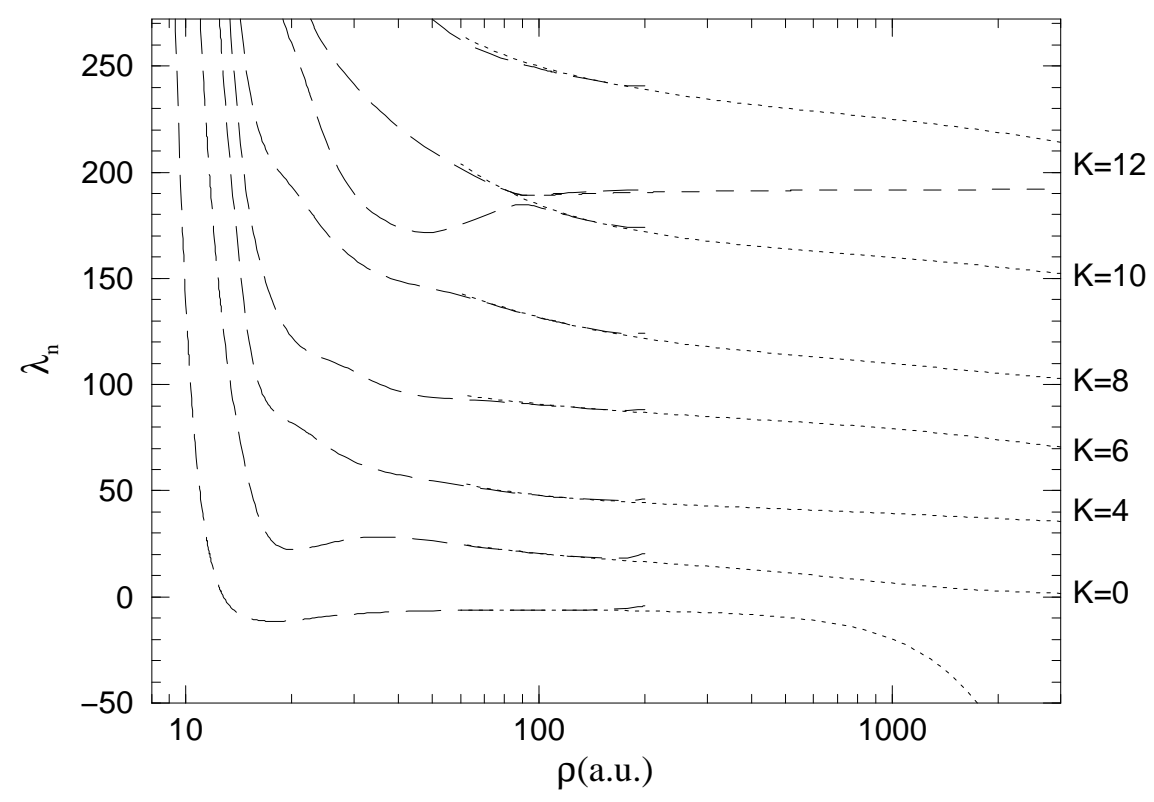

Figure 1: The lowest angular eigenvalues $\lambda_{n}$ for the ${ }^{4}$ He-trimer as functions of $\rho$. The logarithmic scale on the $\rho$-axis uses the atomic length unit. The long-dashed curves are the angular eigenvalues obtained by diagonalization for the relatively small distances, the dotted curves are the large-distance $s$-wave solution and the short dashed curve is the extrapolation of the 7 'th eigenvalue (non-zero $\ell$ ) obtained by using the form $12(12+4)+c \rho^{-2}$. The hyperspherical quantum numbers $K$ are shown to indicate the asymptotic spectrum $K(K+4)$ for $\rho \rightarrow \infty$. The 7'th and 8'th eigenvalues both approach the same asymptotic value $12(12+4)=192$. The lowest eigenvalue diverges as $-\rho^{2}$ corresponding to the bound dimer state. 


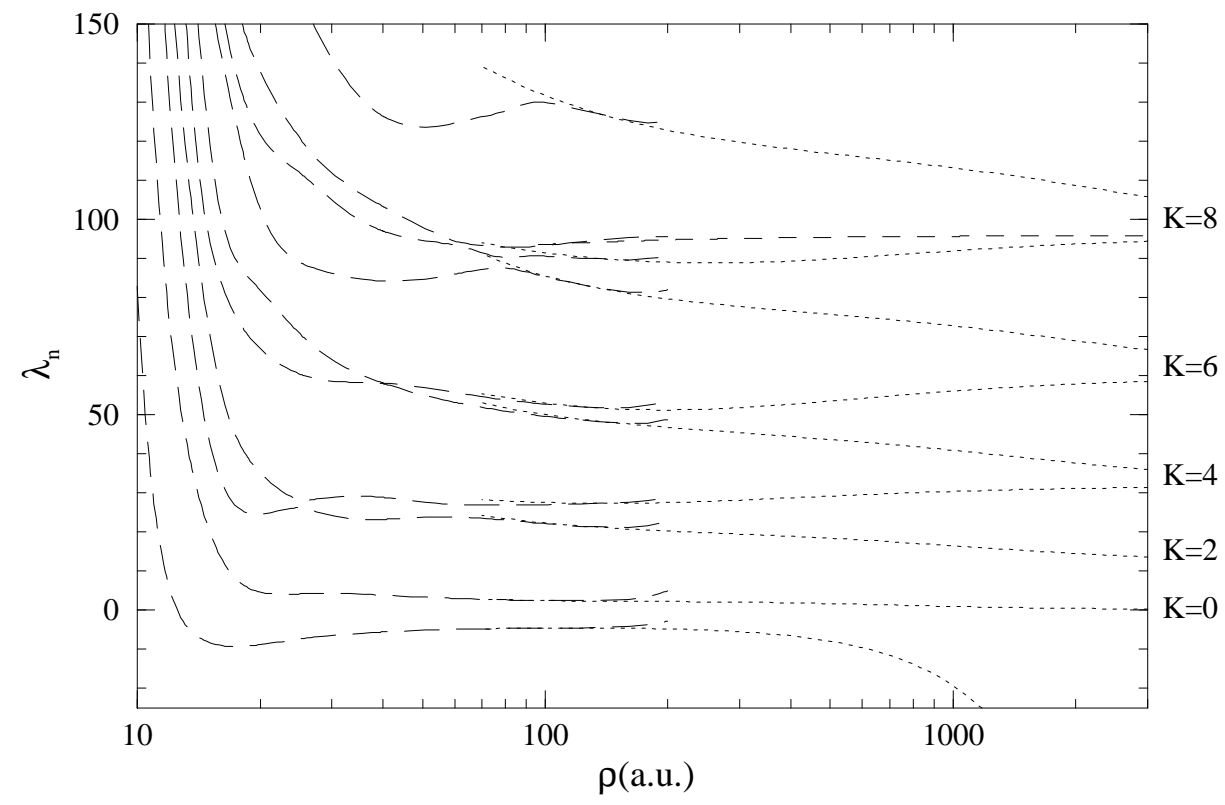

Figure 2: The lowest angular eigenvalues $\lambda_{n}$ for the ${ }^{3} \mathrm{He}^{4} \mathrm{He}_{2}$-trimer as functions of $\rho$. The logarithmic scale on the $\rho$-axis uses the atomic length unit. The long-dashed curves are the angular eigenvalues obtained by diagonalization for the relatively small distances, the dotted curves are the large-distance $s$-wave solution and the short dashed curve is the extrapolation of the 9'th eigenvalue (non-zero $\ell$ ) obtained by using the form $8(8+4)+c \rho^{-2}$. The hyperspherical quantum numbers $K$ are shown to indicate the asymptotic spectrum $K(K+4)$ for $\rho \rightarrow \infty$. The lowest eigenvalue diverges as $-\rho^{2}$ corresponding to the bound dimer state. 


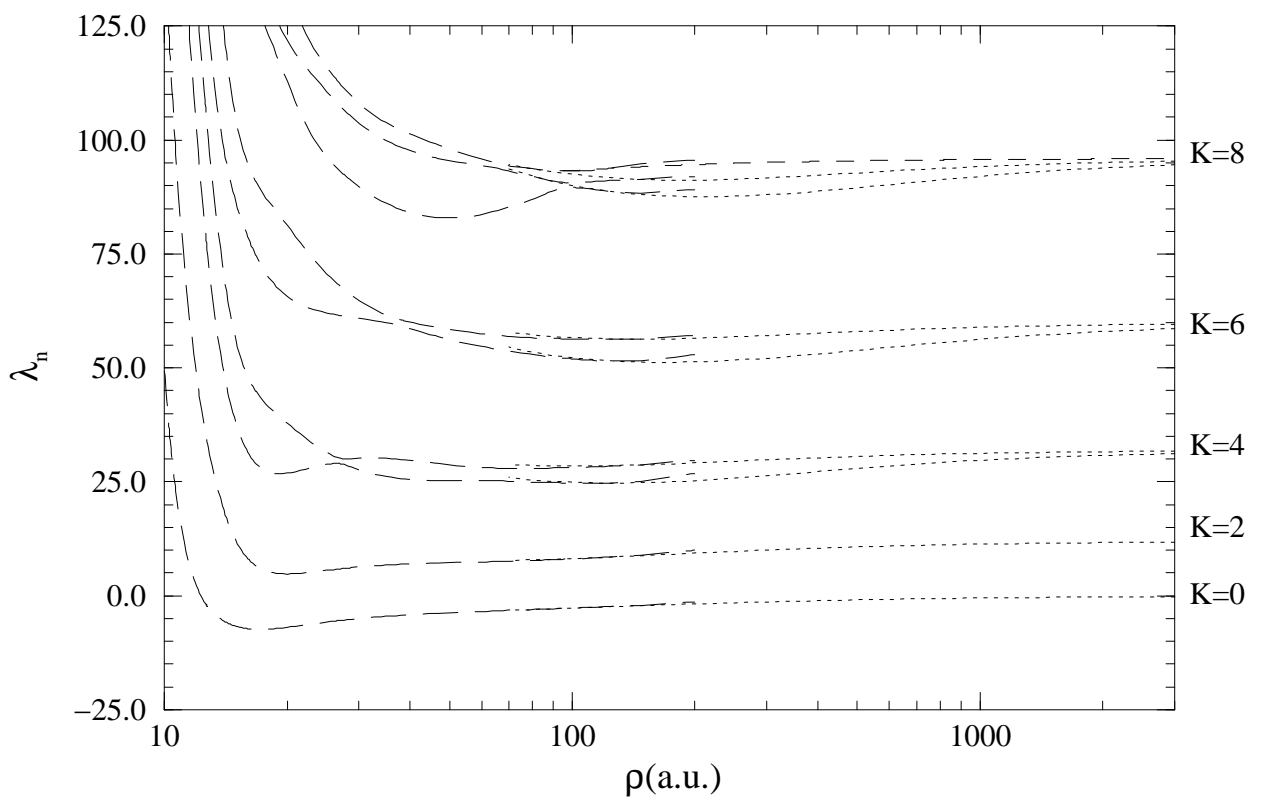

Figure 3: The lowest angular eigenvalues $\lambda_{n}$ for the ${ }^{4} \mathrm{He}^{3} \mathrm{He}_{2}$-trimer as functions of $\rho$. The logarithmic scale on the $\rho$-axis uses the atomic length unit. The long-dashed curves are the angular eigenvalues obtained by diagonalization for the relatively small distances, the dotted curves are the large-distance $s$-wave solution and the short dashed curve is the extrapolation of the 9'th eigenvalue (non-zero $\ell$ ) obtained by using the form $8(8+4)+c \rho^{-2}$. The hyperspherical quantum numbers $K$ are shown to indicate the asymptotic spectrum $K(K+4)$ for $\rho \rightarrow \infty$. The lowest eigenvalue remains finite at large $\rho$.

is the scattering length of the two-body system with particles $j$ and $k$ ), i.e. -2042 a.u., -459 a.u. and 195 a.u., respectively. The different rates are clearly seen in figs. 1, 1 and 3 especially when the range of the plotted $\lambda$-values are considered. The levels all approach their asymptotic values from below, since the sum of scattering lengths in this case is positive, compare to eq.(30) for identical particles for $s$-waves.

\subsection{Energies and wave functions}

For halo states the decisive properties of the potentials are related to the lowenergy scattering behavior or equivalently to the large distance behavior. This is formally expressed in the effective range theory where disparate potentials give the same low-energy results provided they have the same scattering length 
Table 1: Strength and range arameters for gaussian, $V(r)=-S_{0} \exp \left(-r^{2} / b^{2}\right)$, exponential $V(r)=-S_{0} \exp (-r / b)$ and square well $-S_{0} \Theta(r<b)$ potentials for the different He two-body systems. They all have the same scattering length $a_{s}$ and effective range $R_{e f f}$ as the LM2M2 interaction [26], i.e. $a_{s}=-189.054,33.261,13.520$ a.u. and $R_{e f f}=18.564,13.843,25.717$ a.u. for ${ }^{4} \mathrm{He}-{ }^{4} \mathrm{He},{ }^{4} \mathrm{He}-{ }^{3} \mathrm{He},{ }^{3} \mathrm{He}-{ }^{3} \mathrm{He}$, respectively. The strengths are in Kelvin $(1 \mathrm{~K}=$ $0.0861735 \mathrm{meV}=3.16679 \cdot 10^{-6}$ a.u.) and the ranges are in atomic units (1 a.u $=0.524177 \AA)$.

\begin{tabular}{|c||c|c||c|c||c|c|}
\hline \multicolumn{1}{|c||}{ Potential } & \multicolumn{2}{c||}{${ }^{4} \mathrm{He}-{ }^{4} \mathrm{He}$} & \multicolumn{2}{c||}{${ }^{4} \mathrm{He}^{3} \mathrm{He}$} & \multicolumn{2}{c|}{${ }^{3} \mathrm{He}-{ }^{3} \mathrm{He}$} \\
\hline & $S_{0}(K)$ & $b($ a.u. $)$ & $S_{0}(K)$ & $b($ a.u. $)$ & $S_{0}(K)$ & $b($ a.u. $)$ \\
\hline Gaussian & 1.227 & 10.03 & 0.8925 & 10.55 & 0.16186 & 11.27 \\
Exponential & 3.909 & 4.117 & 3.284 & 4.088 & 2.499 & 4.233 \\
Square well & 0.5578 & 14.28 & 0.3691 & 15.61 & 0.2399 & 17.07 \\
\hline
\end{tabular}

and effective range. Since smooth and slowly varying potentials allow easy and accurate computations we adjusted the parameters of a square well $-S_{0} \Theta(r<$ $\left.R_{0}\right)$, a gaussian $V(r)=-S_{0} \exp \left(-r^{2} / b^{2}\right)$ and an exponential potential $V(r)=$ $-S_{0} \exp (-r / b)$ to reproduce $a_{s}$ and $R_{e f f}$ of the LM2M2-potential. The resulting parameters are given in table 1 .

The lowest and decisive eigenvalue for these potentials is plotted in fig. 1 as function of $\rho$. The eigenvalues for all the three simple potentials coincide for $\rho>40$ a.u. whereas that of LM2M2 lies slightly below and slowly approaching the others as $\rho$ increases. The deviations are enhanced due to the focus on one $\lambda$-value and the restriction to distances less than 200 a.u. The differences can only be interpreted as effects of terms of higher order than in the effective range expansion. They are not completely negligible in this region because of the large repulsive core of the LM2M2-potential. At smaller distances the simple potentials produce eigenvalues approaching zero for $\rho=0$. This behavior is then compensated by less attraction in the pocket region.

The binding energies are obtained by solving the coupled set of radial equations. The lower and upper limits can be found by using only the lowest adiabatic potential with and without the diagonal term $Q_{11}$, see 30]. These limits were computed in [8]. We repete this computation using our method and we furthermore increase the number of adiabatic potentials until the results have converged. For the ${ }^{4}$ He-trimer we also find one excited state in agreement with the discussion in connection with eq. (35). One additional oscillation would extend beyond 10 times the scattering length and a third excited state is therefore not possible even 


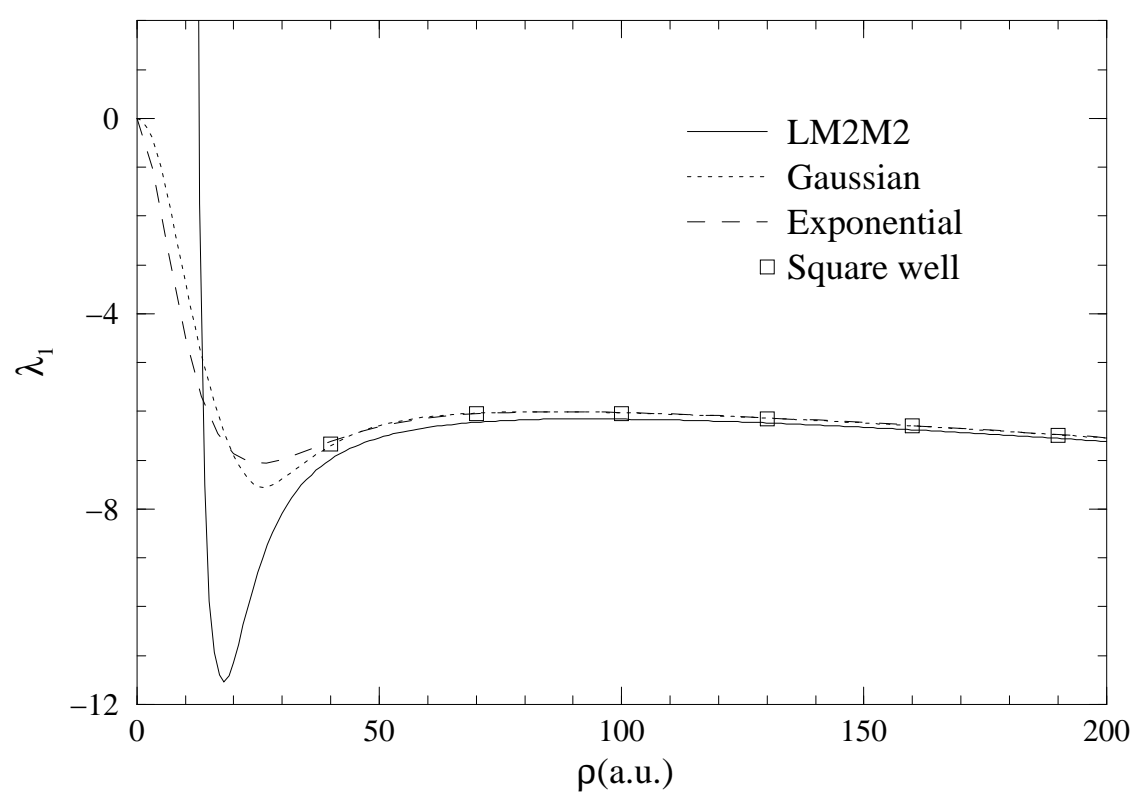

Figure 4: The lowest angular eigenvalue as a function of $\rho$ for the ${ }^{4} \mathrm{He}$-trimer for the realistic LM2M2 [26] potential and the three schematic potentials defined in table 1. The square well results are obtained from eq.(26). 
Table 2: The energies in $\mathrm{mK}$ of the ground state and the excited state of the ${ }^{4}$ He-trimer for one realistic and two schematic potentials. The rows in the table display the numerical results for different numbers of adiabatic channels in the basis. We also give the results of Esry et al. [8] calculated for the LM2M2 potential [26. The row labeled $Q_{11}=0$ displays the results obtained with the lowest adiabatic potential without the diagonal term $Q_{11}$.

\begin{tabular}{|c||c|c||c|c||c|c||c|c|}
\hline \multicolumn{1}{|c||}{ Potential } & \multicolumn{2}{c||}{ Esry et.al [8] } & \multicolumn{2}{c||}{ LM2M2 [26] } & \multicolumn{2}{c||}{ Gaussian } & \multicolumn{2}{c|}{ Exponential } \\
\hline$Q_{11}=0$ & -293.7 & -3.518 & -293.5 & -3.517 & -152.6 & -2.576 & -176.0 & -2.823 \\
\hline 1 & -106.1 & -2.118 & -105.9 & -2.121 & -150.2 & -2.468 & -173.9 & -2.714 \\
2 & & & -119.5 & -2.224 & -150.6 & -2.482 & -174.1 & -2.729 \\
3 & & & -122.0 & -2.245 & -150.7 & -2.485 & -174.2 & -2.731 \\
4 & & & -123.9 & -2.259 & -150.7 & -2.485 & -174.2 & -2.731 \\
5 & & & -124.3 & -2.263 & -150.7 & -2.485 & -174.2 & .2 .732 \\
6 & & & -124.7 & -2.265 & -150.7 & -2.486 & -174.2 & -2.732 \\
7 & & & -125.1 & -2.267 & & & & \\
8 & & & -125.2 & -2.269 & & & & \\
\hline
\end{tabular}

without the $Q_{11}$-term.

We compare in table 2 binding energies for the LM2M2 and the simple interactions for both the ground state and the excited state of the ${ }^{4} \mathrm{He}$-trimer. We confirm the limits computed in [8]. The lowest 7 to 8 adiabatic potentials are needed to obtain the accurate energies deviating from the upper limit by about $20 \%$ and $7 \%$ for the ground and excited states, respectively. This relatively slow convergence for the LM2M2 potential is due to the large repulsive core. Less adiabatic potentials are needed for convergence for the simple two-body interactions, where the lower and upper limits in any case are very close and a larger basis therefore also for this reason is less needed. The binding energies for the gaussian potential are about $20 \%$ and $10 \%$ higher than for the LM2M2 interaction for the ground state and the excited state, respectively. The corresponding numbers for the exponential potential are $40 \%$ and $20 \%$.

The large-distance tails of gaussian and exponential two-body potentials are very different and the variations established here therefore measure the uncertainties inherent in such estimates. These results strongly indicate that such weakly bound states can be studied by simple but carefully chosen potentials provided an accuracy of less than about $40 \%$ and $20 \%$ are required for states located respectively at the edge and far outside the short-range potential. Further accuracy can be obtained with an appropriate choice of radial shape of the simple two-body potential. The computations simplify due to the lack of repulsion at small distances and the smaller basis required. 
Table 3: The energies in $\mathrm{mK}$ of the ground state of the ${ }^{3} \mathrm{He}^{4} \mathrm{He}_{2}$-trimer for one realistic and two schematic potentials. The rows in the table display the numerical results for different numbers of adiabatic channels in the basis. We also give the results of Esry et al. [8] calculated for the LM2M2 potential [26]. The row labeled $Q_{11}=0$ displays the results obtained with the lowest adiabatic potential without the diagonal term $Q_{11}$.

\begin{tabular}{|c||c||c|c|c|}
\hline Potential & Esry et.al [8] & LM2M2 [26] & Gaussian & Exponential \\
\hline \hline$Q_{11}=0$ & -86.52 & -86.47 & -20.44 & -26.06 \\
\hline \hline 1 & -10.22 & -9.682 & -18.41 & -24.27 \\
\hline 2 & & -10.12 & -18.67 & -24.52 \\
\hline 3 & & -11.90 & -18.74 & -24.57 \\
\hline 4 & & -12.74 & -18.79 & -24.60 \\
\hline 5 & & -12.86 & -18.81 & -24.61 \\
\hline 6 & & -13.23 & -18.81 & -24.62 \\
\hline 7 & & -13.34 & -18.81 & -24.61 \\
\hline 8 & & -13.55 & -18.82 & -24.61 \\
\hline 9 & & -13.64 & -18.82 & -24.61 \\
\hline 10 & & -13.66 & -18.82 & -24.61 \\
\hline
\end{tabular}

Substituting one of the ${ }^{4} \mathrm{He}$-atoms in the ${ }^{4} \mathrm{He}$-trimer by a ${ }^{3} \mathrm{He}$-atom decreases the binding energies and only the ground state remains bound. We compare in table 3 the results for different potentials. Unlike the symmetric case we now obtain slightly less binding energy than in [8]. The difference is less than $0.1 \%$ compared to the strengths of the potentials but about $5 \%$ compared to the binding energy itself. Our convergence was thoroughly tested again, but we were not able to find the origin of the discrepancy. As for the symmetric system an accurate binding energy requires 5 to 6 adiabatic potentials for the LM2M2 interaction and again about $40 \%$ is gained compared to the upper limit obtained by using only the lowest adiabatic potential. The simple potentials are now off by factors 1.4 and 1.8 for gaussian and exponential radial shapes, respectively.

The radial wave function has a distribution of components corresponding to the different adiabatic potentials, see eq.(17). The lowest is by far carrying the largest probability as seen in tables 4 and 5 for the two He-trimer systems. The exceedingly small occupation probability for the higher lying angular eigenvalues 
Table 4: Occupation probabilities as functions of adiabatic channels for the ground state (left) and the excited state (right) of the ${ }^{4} \mathrm{He}$-trimer for the three different potentials in table 1 .

\begin{tabular}{|c||c|c||c|c||c|c|}
\hline \multicolumn{1}{|c||}{ Potential } & \multicolumn{2}{c||}{ LM2M2 [26] } & \multicolumn{2}{c||}{ Gaussian } & \multicolumn{2}{c|}{ Exponential } \\
\hline 1 & 0.9978 & 0.9989 & 0.9997 & 0.9991 & 0.9998 & 0.9990 \\
2 & $1.72 \cdot 10^{-3}$ & $1.02 \cdot 10^{-3}$ & $2.61 \cdot 10^{-4}$ & $9.16 \cdot 10^{-4}$ & $1.69 \cdot 10^{-3}$ & $9.8 \cdot 10^{-4}$ \\
3 & $4.0 \cdot 10^{-4}$ & $2.7 \cdot 10^{-5}$ & $2.8 \cdot 10^{-5}$ & $1.9 \cdot 10^{-5}$ & $1.6 \cdot 10^{-4}$ & $1.8 \cdot 10^{-4}$ \\
4 & $8.5 \cdot 10^{-5}$ & $5.5 \cdot 10^{-6}$ & $1.5 \cdot 10^{-6}$ & $4.3 \cdot 10^{-6}$ & $8.9 \cdot 10^{-7}$ & $3.7 \cdot 10^{-5}$ \\
5 & $2.4 \cdot 10^{-5}$ & $2.6 \cdot 10^{-6}$ & $5.9 \cdot 10^{-7}$ & $2.0 \cdot 10^{-6}$ & $3.7 \cdot 10^{-6}$ & $8.4 \cdot 10^{-7}$ \\
6 & $4.6 \cdot 10^{-6}$ & $8.3 \cdot 10^{-7}$ & $1.4 \cdot 10^{-7}$ & $5.8 \cdot 10^{-7}$ & $9.4 \cdot 10^{-8}$ & $2.6 \cdot 10^{-7}$ \\
7 & $1.0 \cdot 10^{-5}$ & $1.2 \cdot 10^{-7}$ & & & & \\
8 & $3.9 \cdot 10^{-6}$ & $3.2 \cdot 10^{-7}$ & & & & \\
\hline
\end{tabular}

seems off hand to contradict the relatively large contribution to the binding energies. The rather instructive explanation for weakly bound systems is that the variation in binding energy might appear to be large when compared to the binding energy itself, but insignificant compared to the strength of the interactions responsible for the binding, see fig. 4 where the potential depths are around 2 $\mathrm{K}$ compared to the binding energies of $\mathrm{mK}$. Thus the lowest adiabatic potentials give a good approximation for the wave functions although the corresponding binding energies only are obtained with less relative accuracy.

The dominating components of the radial wave functions for the ground state and excited state of the ${ }^{4}$ He-trimer are shown in fig. 5 along with the different effective radial potentials. The repulsive core pushes the ground state towards larger distances, whereas the simple potentials allow finite probability also at small distances. The excited state is already at a large distance and therefore less affected by the changing potentials. The wave functions related to the different potentials are quite similar. The length of the first oscillation is about the 350 a.u. as predicted from eq.(34) with $R_{e f f}=18$ a.u. The next would extend 20 times as far, i.e. about 7000 a.u.

\subsection{Geometric structure}

The wave functions in fig. 5 are clearly not revealing directly the geometric structure of these three-body systems. This is not only because the angular part of the 
Table 5: Occupation probabilities as functions of adiabatic channels for the ground state of the ${ }^{3} \mathrm{He}^{4} \mathrm{He}_{2}$-trimer for the three different potentials in table 3 .

\begin{tabular}{|c||c|c|c|}
\hline Potential & LM2M2 & Gaussian & Exponential \\
\hline 1 & 0.9959 & 0.9974 & 0.9979 \\
2 & $3.43 \cdot 10^{-3}$ & $2.50 \cdot 10^{-3}$ & $2.03 \cdot 10^{-3}$ \\
3 & $2.8 \cdot 10^{-4}$ & $1.0 \cdot 10^{-4}$ & $7.5 \cdot 10^{-5}$ \\
4 & $2.4 \cdot 10^{-4}$ & $2.6 \cdot 10^{-5}$ & $1.2 \cdot 10^{-5}$ \\
5 & $6.9 \cdot 10^{-5}$ & $1.6 \cdot 10^{-5}$ & $9.9 \cdot 10^{-6}$ \\
6 & $4.7 \cdot 10^{-5}$ & $5.4 \cdot 10^{-7}$ & $3.0 \cdot 10^{-7}$ \\
7 & $1.9 \cdot 10^{-6}$ & $8.9 \cdot 10^{-7}$ & $5.7 \cdot 10^{-7}$ \\
8 & $1.9 \cdot 10^{-5}$ & $1.2 \cdot 10^{-6}$ & $6.9 \cdot 10^{-7}$ \\
9 & $2.4 \cdot 10^{-6}$ & & \\
10 & $6.1 \cdot 10^{-7}$ & & \\
\hline
\end{tabular}




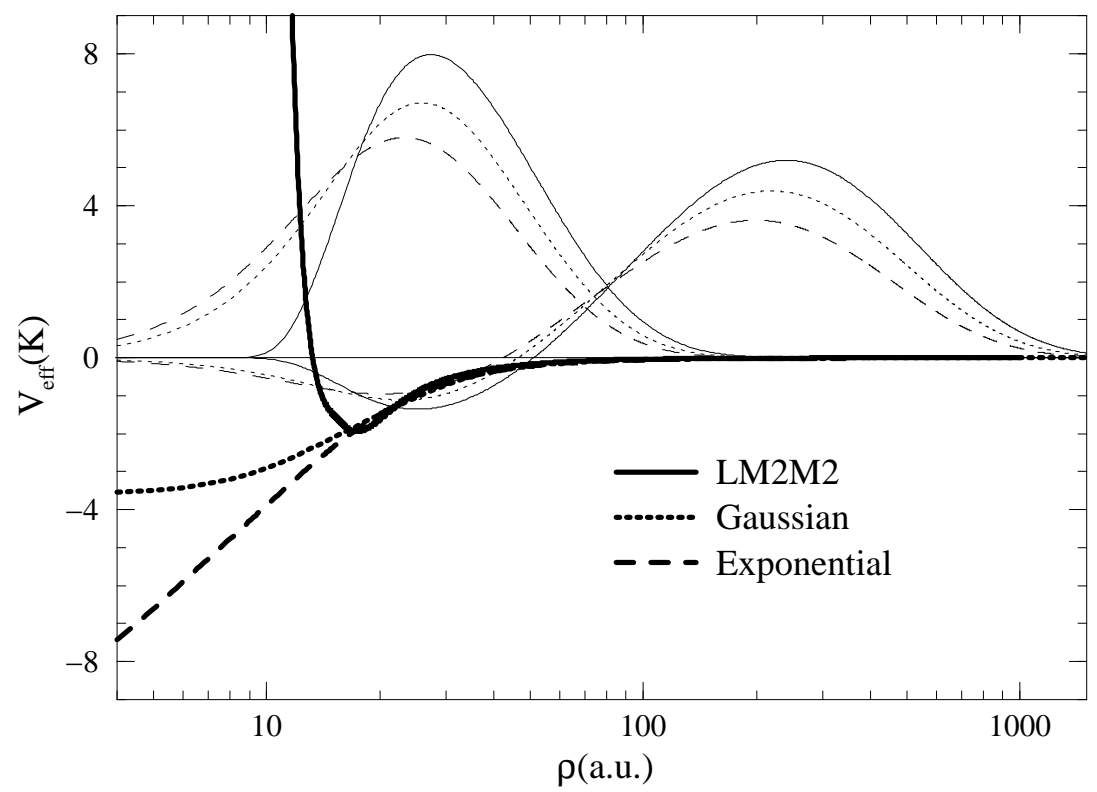

Figure 5: The lowest adiabatic effective radial potentials (thick curves) $V_{\text {eff }}(\rho)=$ $\frac{\hbar^{2}}{2 m}\left(\left(\lambda_{1}+\frac{15}{4}\right) \rho^{-2}-Q_{11}\right)$ and the wave functions corresponding to the two bound states (thin curves) for the ${ }^{4} \mathrm{He}$-trimer for one relalistic [26] and two schematic two-body interactions defined in table 1. The scale on the $\rho$-axis is logarithmic. 

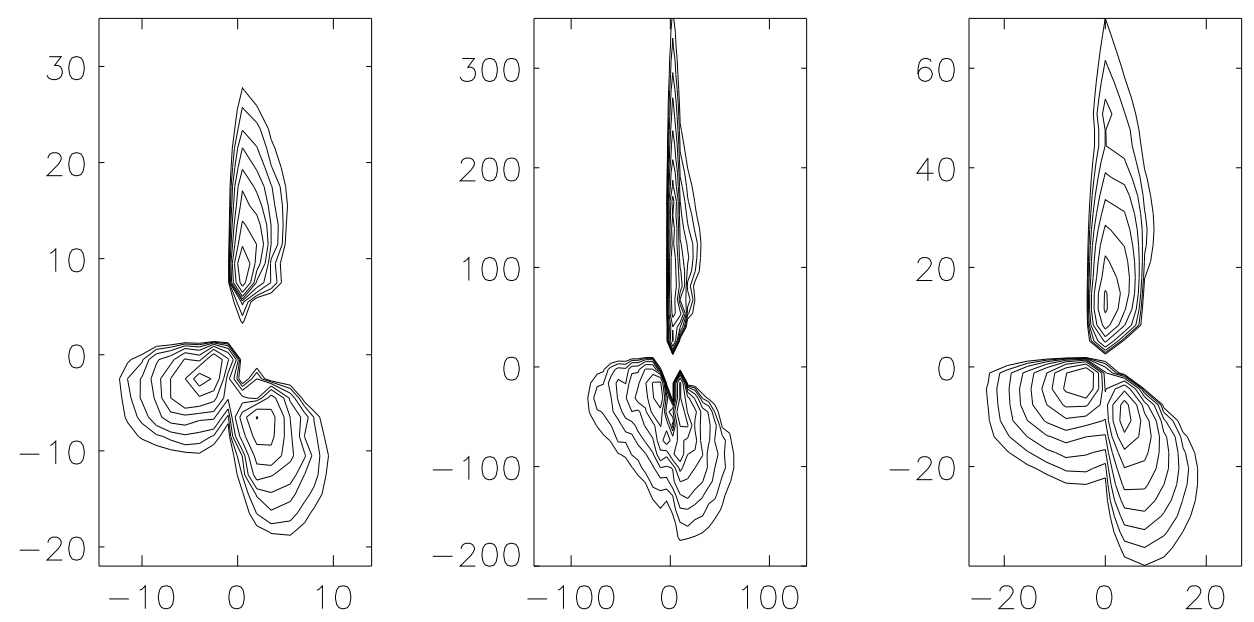

Figure 6: The contour diagrams of the particle density distributions in the intrinsic coordinate system for the ground state (left) and the excitede state of the ${ }^{4}$ He-trimer (middle) and the bound state of ${ }^{3} \mathrm{He}^{4} \mathrm{He}_{2}$ (right). The units on the axes are atomic lengths units. They are different for the three states. There is a factor of 1.5 between each contour curve. The scales on the plots are different.

wave function is missing, but also because the plotted total wave function fully includes all the appropriate symmetries, i.e. symmetric in exchanges of identical bosons and rotational symmetry averaging over all directions. To get a visual picture of the structure we want to consider the plane through the three particles at points $\mathbf{r}_{i}$. The center of mass is at the origin. Let us take the y-axis as the principle axis with the lowest moment of inertia. We furthermore assume that one particle is in the first and two particles are in the third and fourth quadrants. This leaves us with the intrinsic geometry of the trimer.

The resulting contour plots are shown in fig. 6. Note that the distance scales on the individual figures are quite different. The triangular shape for the ${ }^{4} \mathrm{He}-$ trimer ground state (left) is quite apparent. The excited state in the middle show that two of the particles prefer to be close while the third particle is further away. The Efimov conditions are almost fulfilled, i.e. very large scattering lengths for at least two of the binary subsystems. As we shall discuss later this figure illustrates the structure of an Efimov state. Any higher-lying Efimov state could simply be found by a scaling of the axes, see eq.(31).

The contour diagram at the right side of fig. 6 also show the preference for two relatively close-lying particles. However, only one scattering length is now large and the Efimov conditions are not fulfilled. It is instructive to imagine that the ${ }^{3} \mathrm{He}-{ }^{4} \mathrm{He}$ scattering length is increased and the subsequent appearence of the 

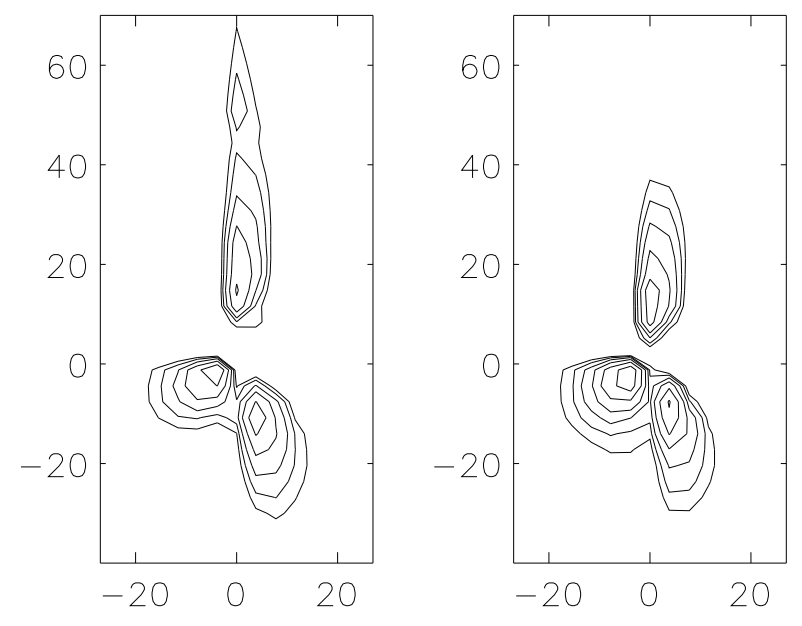

Figure 7: The contour diagram of the ${ }^{3} \mathrm{He}$ density (left) and half of the ${ }^{4} \mathrm{He}$ density distribution (right) in the intrinsic coordinate system for the bound state of ${ }^{3} \mathrm{He}^{4} \mathrm{He}_{2}$ (right). The units on the axes are atomic lengths. There is a factor of 1.5 between each contour. Both the scale and the contours are the same as on the total density distribution in figure 6 .

Efimov states. The lowest eigenvalue in fig. 2 would still bend over and diverge due to the bound state. The second eigenvalue would approach a negative constant above the lowest eigenvalue. The corresponding adiabatic potential would isolated provide the Efimov states. However, these states would appear in the continuum as excited states above the ground state shown in the figure. Thus the ground state of the asymmetric system cannot be an approximate Efimov state. The superficial resemblance of the middle and right figures therefore illustrates how deceiving the density distribution can be. Additional information is needed to come up with the correct interpretation.

For the asymmetric system we can also compute the individual particle density as shown in fig. 7. The left figure can be obtained from the right figure if a small component with an elongated distribution is added. The ${ }^{4}$ He-trimer appears in a completely symmetric state while the asymmetric system in addition also has a relatively small asymmetric component of the ${ }^{3} \mathrm{He}-{ }^{4} \mathrm{He}$ relative wave function. The large symmetric component resembles the ${ }^{4} \mathrm{He}$-trimer and the small asymmetric configuration, which becomes dominating at larger distances where the structure of the ${ }^{4} \mathrm{He}$-dimer is approached.

The triangular structure at the left side of fig. 6 is apart from the scale similar to the three-body cluster approximation of the ground state of ${ }^{12} \mathrm{C}$, whereas the 
two other trimer states are very different from the almost linear configuration of the astrophysically interesting first excited $0^{+}$-state of ${ }^{12} \mathrm{C}$, which therefore is far from being an Efimov state, see [27].

The geometry of the He-trimers can be studied quantitatively by computation of various expectation values related to the distances between the particles. We first consider the sizes shown in table 6 where two measures are used, i.e. the root mean square radius and the (first order) distance from the center of mass. The dimer has an average distance between the particles of about 100 a.u., which is at the lower end of the experimentally allowed range of $120 \pm 20$ a.u. [22]. The three-body ground state is much smaller than the ${ }^{4}$ He-dimer [8, 22]. However, the size of about $100 \mathrm{a} . \mathrm{u}$. of the excited state is around $50 \%$ larger than for the dimer both when measured by the first and the second moment. This radius is larger than the range of the effective two-body interaction but smaller than the size (189 a.u.) of the scattering length. The spatial extension of the probability reaches beyond $\left|a_{s}\right|$ as seen in fig. 5, but the wave function is still well within the range of the effective radial three-body potential ( $\rho^{-2}$ behavior) extending to about $\left|15 \mu_{i j} a_{s}\right|$, see eq.(30). These proportions are characteristic for an Efimov state.

The ratio between the three-body radii of excited and ground state is 9.5 which only is two times smaller than the asymptotic prediction from eq.(34). In comparison the ratio of the binding energies is 52 which is about 7 times smaller than predicted in eq.(36) for two Efimov states. These observations simply confirm that the ground state does not have the characteristics of an Efimov state. An additional (second) excited state (which is impossible for this scattering length) would have obeyed these scaling laws for radii and energies for Efimov states.

The asymmetric system ${ }^{3} \mathrm{He}^{4} \mathrm{He}_{2}$ has only one bound state with both binding energy and size in between the corresponding values for the ground state and the excited state of the symmetric system, see tables 2, 3 and 6. For Efimov states the product of the energy and the square of the radius should be a constant, see eqs.(34) and (36). This product is four times larger for the excited symmetric state than for the asymmetric state. The radius should then be about twice as large if the state should resemble an Efimov state. However, this seems to be unlikely, since the radius is of the same order as the two-body effective ranges. In any case the interpretation as an approximate Efimov state was already excluded in the discussion above. Thus a pronounced halo structure is therefore a more appropriate description.

The distance between the two ${ }^{4}$ He-particles computed as $\left\langle\left|\mathbf{r}_{1}-\mathbf{r}_{2}\right|\right\rangle$ is 28 a.u. and the distance between ${ }^{3} \mathrm{He}$ and one specific of the ${ }^{4} \mathrm{He}$-particles is 38 a.u. The distances between both the ${ }^{3} \mathrm{He}-{ }^{4} \mathrm{He}$ and the two ${ }^{4} \mathrm{He}$ particles are then two to three times smaller in this asymmetric bound state than in the bound ${ }^{4} \mathrm{He}$ dimer. As necessary both distances are in between the minimum and maximum of 20 and 46 a.u. Again this demonstrates that the bound dimer-state is not a dominating configuration in the three-body systems. 
Table 6: Expectation values of various operators related to the geometrical structure of the bound states of the He-dimer and the He-trimers. The results for the different potentials in tables 2 and 5 are given for each quantity: LM2M2 (top), gaussian (middle) and exponential (bottom). The coordinate of particle $i$ is $\mathbf{r}_{i}$, the center of mass coordinate is $\mathbf{R}$ and $n=2,3$ is the number of particles. All the lengths are in atomic units.

\begin{tabular}{|c|c|c|c|c|}
\hline & ${ }^{4} \mathrm{He}_{2}$ & ${ }^{4} \mathrm{He}_{3}$ & ${ }^{4} \mathrm{He}_{3}^{*}$ & ${ }^{3} \mathrm{He}^{4} \mathrm{He}_{2}$ \\
\hline$\sqrt{\left\langle\sum_{i}\left(\mathbf{r}_{i}-\mathbf{R}\right)^{2}\right\rangle / n}$ & 66.9 & 11.8 & 115 & 26 \\
& 66.9 & 10.8 & 104 & 22 \\
& 66.9 & 9.9 & 95 & 20 \\
\hline$\left.\sum_{i}\left|\mathbf{r}_{i}-\mathbf{R}\right|\right\rangle / n$ & 49.0 & 10.2 & 96 & 20 \\
& 49.0 & 9.4 & 86 & 17.5 \\
& 49.0 & 8.6 & 77 & 15.6 \\
\hline $\left.\min _{i \neq j}\left|\mathbf{r}_{i j}\right|\right\rangle$ & 98.1 & 11.8 & 70 & 20 \\
& 98.0 & 10.6 & 67 & 19.1 \\
\hline $\left.\max _{i \neq j}\left|\mathbf{r}_{i j}\right|\right\rangle$ & 98.0 & 9.6 & 64 & 17.0 \\
\hline$\left\langle\frac{\min _{i \neq j}\left|\mathbf{r}_{i j}\right|}{\max _{i \neq j}\left|\mathbf{r}_{i j}\right\rangle}\right.$ & 98.1 & 23 & 219 & 46 \\
& 1 & 21 & 195 & 40 \\
& 1 & 0.51 & 0.37 & 0.49 \\
& 19.0 & 19.3 & 178 & 35 \\
\hline
\end{tabular}


Another interesting quantity in table 6 is the expectation value of the ratio between the smallest and the largest interparticle distance. First the minimum and maximum average distances themselves differ by a factor of two for the ground states and a factor of three for the excited Efimov-like state. The ratio is nearly 0.5 for both ground states and 0.34 for the excited state. If the angular part (including the $\alpha$ coordinate) of the wave function was constant this number would be 0.52. In the case of an Efimov state this number was computed to be 0.38. Thus the excited state does also in this respect resemble an Efimov state. That the ratio is less than the value for an Efimov state indicates nonnegligible contributions corresponding to two particles in a bound state and the third particle further away. This is consistent with the observation that the excited stated is larger than the dimer.

In all cases the simple potentials produce sizes deviating about $10 \%$ and $20 \%$ for gaussian and exponential potentials from those of the LM2M2 interaction. Thus if only this accuracy is required the corresponding simpler computations are sufficient.

\section{Summary and conclusions}

Spatially extended three-body halo states are apparently present in some of the atomic helium-trimer systems. The ${ }^{4} \mathrm{He}$-dimer is experimentally established as exceedingly large and as a consequence it must also be very loosely bound. The large distances are then essential in any accurate description. The possibility of finding Efimov states is adding interest in obtaining a quantitative understanding, but unfortunately at the same time emphasizing the practical difficulties. The necessary large-distance correlations must be treated very accurately without loosing the precision at smaller distances where the binding energy originates. This three-body problem is of quantum mechanical nature since the probability almost entirely is found in the classically forbidden region.

We incorporate the correlations by solving the coordinate space Faddeev equations. We expand the wave function in a complete set of angular eigenfunctions obtained by solving the angular part of the Faddeev equations for a given hyperradius. This adiabatic basis is related to a set of effective radial potentials. The corresponding radial wave functions are solutions to the coupled set of radial differential equations. We first formulate this prescription in general and then the crucial large distances are specifically considered. Only $s$-waves couple at large distances and this fact is exploited to derive simple equations with almost analytic solutions. A suitable numerical procedure follows from this formulation. For exploratory calculations we furthermore suggest a simplifying approximation where the "correct" potential is substituted by almost schematic potentials with relatively little loss of accuracy for the spatially extended states. 
The lowest effective radial potential is decisive and we describe a series of its large-distance analytic properties for a symmetric system. When the two-body scattering length is sufficiently large the Efimov states appear as solutions. We repete the occurrence conditions and a few of the analytic properties of these exotic states.

The strongly repulsive core is necessary in a correct description of the twobody potential. The numerical procedure is somewhat delicate and a sizable basis is necessary. Each of the Faddeev components is expanded on the hyperspherical basis. We need relative angular momentum quantum numbers up to about 10 , Jacobi polynomials for $s$-waves up to order 150 and significantly fewer for the higher partial waves. This numerical procedure produces the solutions at small distances up to about 100 a.u. and from then on we use the solutions obtained directly from the decoupled large-distance set of equations.

The schematic two-body potentials are parametrized to reproduce the scattering length and effective range of the original complicated potential. We use radial shapes of gaussian, exponential and square well form. The solutions are much easier to compute due to the lack of hard core repulsion. Still these simple attractive potentials reproduce the results of the "correct" calculations within $40 \%$ and significantly better for the spatially extended states. More suited shapes could certainly reduce this uncertainty, which in fact is remarkable for several reasons. First this demonstrates the importance of the large-distance behavior of the effective radial potential. Second the error in binding energy by using such simple potentials is roughly the same as the results obtained by using only the lowest adiabatic potential, which carries more than $99 \%$ of the probability in a fully converged calculation. Thus simple potentials could be exploited to gain even semi quantitative insight.

We have essentially confirmed the calculations of [8] using the lowest adiabatic potential. In our full calculations we find additional binding of $7 \%$ to $40 \%$ measured relative to the binding energy, which is several orders of magnitude smaller than the strength of the attraction. Indeed these states are very weakly bound. We investigated the geometry of the bound He-trimer states. The ground state of ${ }^{4} \mathrm{He}_{3}$ is an equal sided triangle. The excited state of ${ }^{4} \mathrm{He}_{3}$ and the ground state of the asymmetric ${ }^{3} \mathrm{He}^{4} \mathrm{He}_{2}$ system are both also triangular but with sides differing by a factor of two to three.

The excited state of ${ }^{4} \mathrm{He}_{3}$ has all the characteristics of an Efimov state whereas the somewhat similar density distribution of the asymmetric state rather should be characterized as a pronounced halo state. More Efimov states would only appear in the symmetric system when the scattering length is increased by about one order of magnitude. Smaller changes would be needed to promote the halo state to the giant halo state called an Efimov state.

In conclusion, we have described a method to compute spatially extended three-body structures. The essential large-distance behavior is specially treated. We apply the method to the atomic helium trimers. The binding energies are 
accurately determined and the geometric structures are found to be triangular configurations. The ground state of ${ }^{4} \mathrm{He}_{3}$ has a triangular shape and the excited state is an Efimov state whereas the ${ }^{3} \mathrm{He}^{4} \mathrm{He}_{2}$ bound state is a halo state.

\section{References}

[1] K. Riisager, A.S. Jensen and P. Møller, Nucl. Phys. A548, 393 (1992).

[2] D.V. Fedorov, A.S. Jensen, and K. Riisager, Phys. Rev. C49, 201 (1994); C50, 2372 (1994); C51, 3052 (1995).

[3] J. Goy, J.-M. Richard and S. Fleck, Phys. Rev. A52, 3511 (1995).

[4] J.-M. Richard and S. Fleck, Phys. Rev. Lett. 73, 1464 (1994).

[5] P.G. Hansen, A.S. Jensen and B. Jonson, Ann. Rev. Nucl. Part. Sci. 45, 591 (1995).

[6] I. Tanihata, J. Phys. G22, 157 (1996).

[7] K. Riisager, Rev. Mod. Phys. 66, 1105 (1994).

[8] B.D. Esry, C.D. Lin and C.H. Greene, Phys. Rev. A54, 394 (1996).

[9] V.M. Efimov, Sov. J. Nucl. Phys. 12, 589 (1971); Phys. Lett. B33, 563 (1970); Comm. Nucl. Part. Phys. 19, 271 (1990).

[10] M.V. Zhukov, B.V. Danilin, D.V. Fedorov, J.M. Bang, I.J. Thompson, and J.S. Vaagen, Phys. Rep. 231, 151 (1993).

[11] J.-M. Richard, Phys. Reports 212, 1 (1992).

[12] C.D. Lin, Phys. Reports 257, 1 (1995).

[13] T. Misu, W. Nazarewics and S. Åberg, Nucl. Phys. A614, 44 (1997).

[14] D.V. Fedorov, A.S. Jensen and K. Riisager, Phys. Lett. B312, 1 (1993).

[15] A. Cobis, D.V. Fedorov and A.S. Jensen, J. Phys. G23, 401 (1997).

[16] R.A. Aziz and M.J. Slaman, J. Chem. Phys. 94, 8047 (1991).

[17] D. V. Fedorov and A. S. Jensen, Phys. Rev. Lett. 71, 4103 (1993).

[18] A. Cobis, D.V. Fedorov and A.S. Jensen, Phys. Rev. Lett. 79, 2411 (1997).

[19] A.S. Jensen, E. Garrido and D.V. Fedorov, Few-Body Systems, 22, 193 (1997). 
[20] D.V. Fedorov, A.S. Jensen and K. Riisager, Phys. Rev. Lett. 73, 2817 (1994).

[21] W. Schöllkopf and J.P. Toennies, J. Chem. Phys 104, 1155 (1996).

[22] F. Lou, C.F. Giese, and W.R. Gentry, J. Chem. Phys 104, 1151 (1996).

[23] L.H. Thomas, Phys. Rev. 47, 903 (1935).

[24] T. Cornelius and W. Glöckle, J. Chem. Phys. 85, 3906 (1986).

[25] B.D. Esry, C.H. Greene, Y Zhou and C.D. Lin, J.Phys. B29, L51 (1996).

[26] R.A. Aziz and M.J. Slaman, J. Chem. Phys. 94, 8047 (1993).

[27] D. V. Fedorov and A. S. Jensen, Phys. Lett. B389, 631 (1996).

[28] R.A. Aziz, V.P.S. Nain, K.S. Carley, W.L. Taylor and G.T. McConville, J. Chem. Phys. 70, 4330 (1979).

[29] J. Raynal and J. Revai, Nuovo Cimento, vol LXVIII, 612 (1970).

[30] L. Spruch, Abstracts of the Int. Conf. Physics of Electrons and Atomic Collisions, Leningrad, 104 (1967).

[31] C.A. Parish and C.E. Dykstra, J. Chem. Phys. 101, 7618 (1994); I. Roeggen and J. Almlöf, J. Chem. Phys. 102, 7095 (1995). 